\title{
Modulating visuomotor sequence learning by repetitive transcranial magnetic stimulation: what do we know so far?
}

Laura Szücs-Bencze ${ }^{1}$, Teodóra Vékony ${ }^{2}$, Orsolya Pesthy ${ }^{3,4}$, Nikoletta Szabó ${ }^{1}$, Zsigmond Tamás Kincses ${ }^{1,5}$, Zsolt Turi* 6 , Dezso Nemeth* 2,4,7

(1) Department of Neurology, University of Szeged, Semmelweis utca 6., H-6725, Szeged, Hungary.

(2) Lyon Neuroscience Research Center (CRNL), INSERM, CNRS, Université Claude Bernard Lyon 1, 95 Boulevard Pinel, 69675 Bron, France.

(3) Doctoral School of Psychology, ELTE Eötvös Loránd University, Izabella utca 46., H-1064, Budapest, Hungary.

(4) Institute of Psychology, ELTE Eötvös Loránd University, Izabella utca 46., H-1064, Budapest, Hungary.

${ }^{(5)}$ Department of Radiology, University of Szeged, Semmelweis utca 6., H-6725, Szeged, Hungary.

(6) Department of Neuroanatomy, Institute of Anatomy and Cell Biology, Faculty of Medicine, University of Freiburg, Freiburg, Germany.

(7) Brain, Memory and Language Research Group, Institute of Cognitive Neuroscience and Psychology, Research Centre for Natural Sciences, Hungarian Academy of Sciences, Budapest, Hungary.

Address correspondence to Teodóra Vékony, Lyon Neuroscience Research Center (CRNL), INSERM, CNRS, Université de Lyon, Centre Hospitalier Le Vinatier - Bâtiment 462 - Neurocampus 95 boulevard Pinel, 69675 Bron, France. E-mail: teodora.vekony@univ-lyon1.fr.

\footnotetext{
* These authors have contributed equally to this work.
} 


\begin{abstract}
Sequence learning and statistical learning are key components of predictive processes and many cognitive, motor, and social skills. The Serial Reaction Time Task (SRTT) can measure this fundamental cognitive process in the visuomotor domain. Repetitive transcranial magnetic stimulation (rTMS) is an increasingly used non-invasive brain stimulation method that can help us to determine the functional role of a given brain region. In this literature review, we systematically analyzed the eligible records $(n=17)$ that sought to modulate the performance on the SRTT with rTMS. The purpose of the analysis was to determine the effects of the following factors on SRTT performance: (1) stimulated brain areas, (2) rTMS protocols, (3) stimulated hemisphere, (4) timing of the stimulation, (5) SRTT sequence properties, and (6) other methodological features. We found that the two most promising target areas are the primary motor cortex (M1) and the dorsolateral prefrontal cortex (DLPFC). Low-frequency protocols over the M1 usually weakened performance, but the results are less consistent for the DLPFC. Our analysis of these six factors could help design future studies to modulate sequence learning by non-invasive brain stimulation.
\end{abstract}

\title{
Keywords
}

non-invasive brain stimulation, sequence learning, statistical learning, repetitive TMS, DLPFC 


\section{Introduction}

Sequence learning is a fundamental ability of the human brain. It forms the basis of many cognitive, social, and motor skills (Bergstrom et al., 2012; Lieberman, 2000; Nemeth et al., 2011). Acquiring the rules of a language, learning to play an instrument, recognizing the habits of a loved one - all occur by becoming sensitive to sequences of events. The Serial Reaction Time Task (SRTT) is one of the most popular paradigms in visuomotor sequence learning research; it has been used in $\sim 2500$ different experimental psychology, clinical and cognitive neuroscience studies.

Previous studies have used observational and interventional methods to better understand the functional role of brain regions and networks that underly sequence learning. On the one hand, neuroimaging studies revealed that particular brain areas engage during the learning and retrieval of sequence information. These include the primary motor cortex (M1), supplementary motor area (SMA), dorsolateral prefrontal cortex (DLPFC), and parietal cortex (Daselaar et al., 2003; Keele et al., 2003; Poldrack et al., 2005; Seidler et al., 2005; Willingham et al., 2002). Whereas neuroimaging methods allow characterizing the spatio-temporal features of neuronal activity, interventional methods can modulate brain activity (e.g., cortical excitability) that can facilitate drawing causal inferences about the functional role of brain regions and networks (Bergmann \& Hartwigsen, 2020). Repetitive transcranial magnetic stimulation (rTMS) is an increasingly used non-invasive brain stimulation (NIBS) method to examine the functional role of cortical areas and brain networks (Bergmann \& Hartwigsen, 2020; Pascual-Leone et al., 1991).

But can rTMS protocols effectively alter sequence learning performance in humans? If yes, which stimulation parameters govern its efficacy? In this paper, we reviewed those studies that examined the cognitive effects of rTMS on visuomotor sequence learning using the SRTT. 
We summarized the existing results and outlined those factors that should be considered when designing a new rTMS-SRTT study.

\section{Different variations of the SRTT}

In the classical SRTT (Nissen \& Bullemer, 1987), the participant must respond to a visual stimulus that appears in one of four horizontal locations by pressing the corresponding button on a response device as quickly and accurately as possible. Unknown to the participant, the stimuli follow each other in a predetermined order, forming a repeating sequence (e.g., "2-3-14-3-2-4-1-3-4-2-1", where numbers represent the four horizontal locations). In the control task, the elements appear in random positions. Compared to the random sequences, participants respond gradually faster and with higher accuracy in the repeating sequences throughout learning, which shows the acquisition of the sequence order.

This classical version of the SRTT measures the implicit learning of a deterministic sequence (Robertson, 2007). In the last few decades, several new versions of this task have emerged, which can differ in three fundamental dimensions: 1) whether the participants are aware of the sequence structure or not (explicit or implicit task), 2) whether an element of the sequence predicts the next element with $100 \%$ or with a certain probability (deterministic or

probabilistic sequence), and 3) whether the preceding or the n-2 element predicts the occurrence of a given element of the sequence (first-order conditional or second-order conditional sequence). As these diverse sequence types assume different cognitive processes (Prashad et al., 2021; Song et al., 2007), their acquisition may also rely on distinct neural networks (Peigneux et al., 2001; Walker \& Stickgold, 2005).

The SRTT performance can be assessed via accuracy or response time (RT) measures. These two measures presumably reflect different processes. In general, RT indicates automatic, habitual processes, while accuracy might reflect more controlled, goal-directed processes 
(Keramati et al., 2011). Participants can achieve SRTT with high accuracy rates throughout the task and most studies report RT-based rather than accuracy-based results. Due to these reasons, we will refer to RT-based results when discussing enhanced or weakened performance in the context of the behavioral effects of rTMS, unless stated otherwise.

\section{Different rTMS protocols}

Unlike single-pulse TMS, rTMS can exert lasting aftereffects on cortical excitability (Fitzgerald et al., 2006; Huang et al., 2017; Klomjai et al., 2015) and is widely used for studying the neural mechanisms of various cognitive processes (Ferrari et al., 2018; Kim et al., 2004; Ruitenberg et al., 2014; Smalle et al., 2017; Verwey et al., 2002). We can distinguish between conventional and patterned rTMS protocols (see Figure 1). Conventional rTMS protocols use a single frequency (e.g., $1 \mathrm{~Hz}, 5 \mathrm{~Hz}, 10 \mathrm{~Hz}$ ) and the temporal pattern of the pulse sequence (i.e., the number of pulses per burst, inter-burst interval, the total number of pulses) can vary substantially across studies. For example, one study may deliver $1 \mathrm{~Hz}$ rTMS as a single, uninterrupted train of 900 pulses (e.g., Bagnato et al., 2005), whereas another one may deliver 300 pulses per burst three times with 60 -second-long inter-burst intervals (e.g. Chouinard et al., 2005).

Patterned rTMS protocols, such as theta-burst stimulation (TBS) standardize many of the stimulation parameters (Huang et al., 2005). TBS protocols consist of bursts that comprise three pulses at $50 \mathrm{~Hz}$ that are repeated at $5 \mathrm{~Hz}$. The most common TBS protocol delivers 600 pulses in total, although shorter (i.e., 300 pulses) or longer (i.e., 1800 pulses) variants also exist (Gentner et al., 2008; McCalley et al., 2021). Based on the presence or absence of intermittent stimulation-free periods, we further distinguish between continuous TBS (cTBS) and intermittent TBS (iTBS) (Huang et al., 2005). In iTBS, the 2-second-long bursts are interrupted 
by 8 -second-long stimulation-free periods, whereas in cTBS, the bursts are delivered continuously.

Based on the induced direction of change in the corticospinal excitability, rTMS is commonly classified according to the frequency of stimulation. Low-frequency rTMS $(\leq 1 \mathrm{~Hz})$ tends to decrease, whereas high-frequency $\operatorname{rTMS}(\geq 5 \mathrm{~Hz})$ is supposed to increase the corticospinal excitability (Dayan et al., 2013; Huang et al., 2017; Polanía et al., 2018). Besides, the effect of rTMS depends on several other stimulation parameters, i.e., stimulation intensity, number of delivered pulses, coil orientation, and the temporal pattern of the protocol (Lang et al., 2006; Pell et al., 2011). It is generally accepted that cTBS tends to decrease, whereas iTBS may increase the corticospinal excitability level (Cárdenas-Morales et al., 2010; Huang et al., 2005; c.f. Hamada et al., 2013).

\section{Conventional}

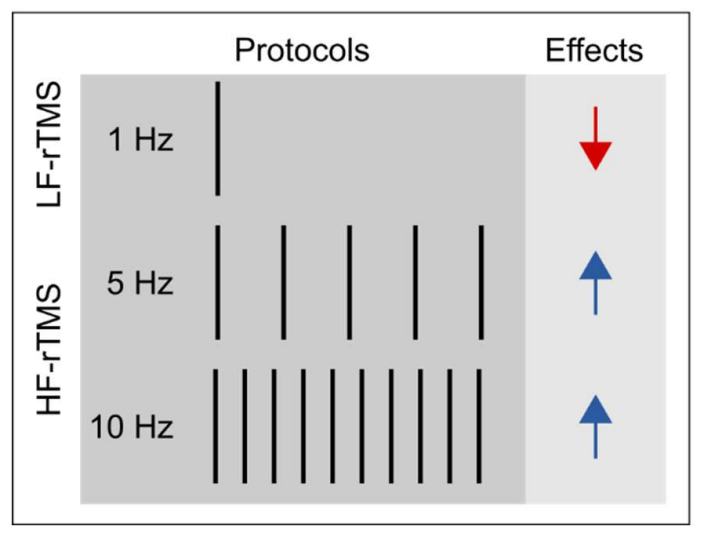

Patterned

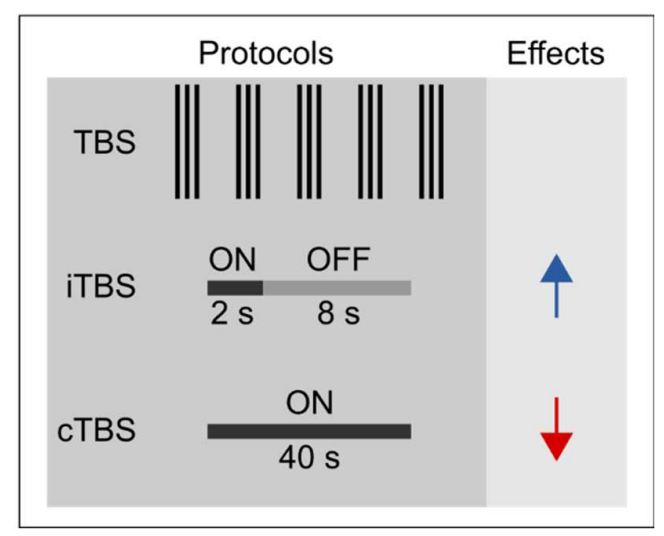

Figure 1. Conventional and patterned rTMS protocols and their assumed aftereffects on corticospinal excitability in the M1. Black bars represent the number of delivered rTMS pulses in one second. Red arrows indicate a decrease, whereas blue arrows indicate an increase in corticospinal excitability. Abbreviations: TBS: theta-burst stimulation; cTBS: continuous TBS; iTBS: intermittent TBS; HF-rTMS: high-frequency rTMS; LF-rTMS: lowfrequency rTMS. 


\section{Literature search and study selection}

We used the Web of Science database for the literature search, which was closed on February $1^{\text {st }}, 2021$. We used the following search syntax: ('sequence learning' OR 'statistical learning' OR 'procedural learning' OR 'procedural knowledge' OR 'implicit learning' OR 'implicit memory' OR 'motor learning' OR 'motor skill' OR 'serial reaction time task') AND ('TMS' OR 'rTMS' OR 'TBS' OR 'cTBS' OR 'iTBS). This search syntax returned 374 hits. We reviewed these results based on their title and abstract and selected 42 articles that seemed relevant, irrespective of publication year or status (see Figure 2).

The inclusion criterion was the use of rTMS together with the SRTT on healthy human participants. Thirty-two of the 42 articles were excluded: 27 of them because they used another sequence learning paradigm (finger tapping task, discrete sequence production task, continuous tracking task); four of them because they used another NIBS technique (paired associative stimulation, transcranial direct current stimulation, static magnetic stimulation); and one study because it examined the effect of rTMS on the SRTT performance of patients with major depressive disorder. Based on the references of the screened articles, we conducted an additional search and found seven other relevant articles; therefore, finally, a total of 17 studies were selected for this review. 


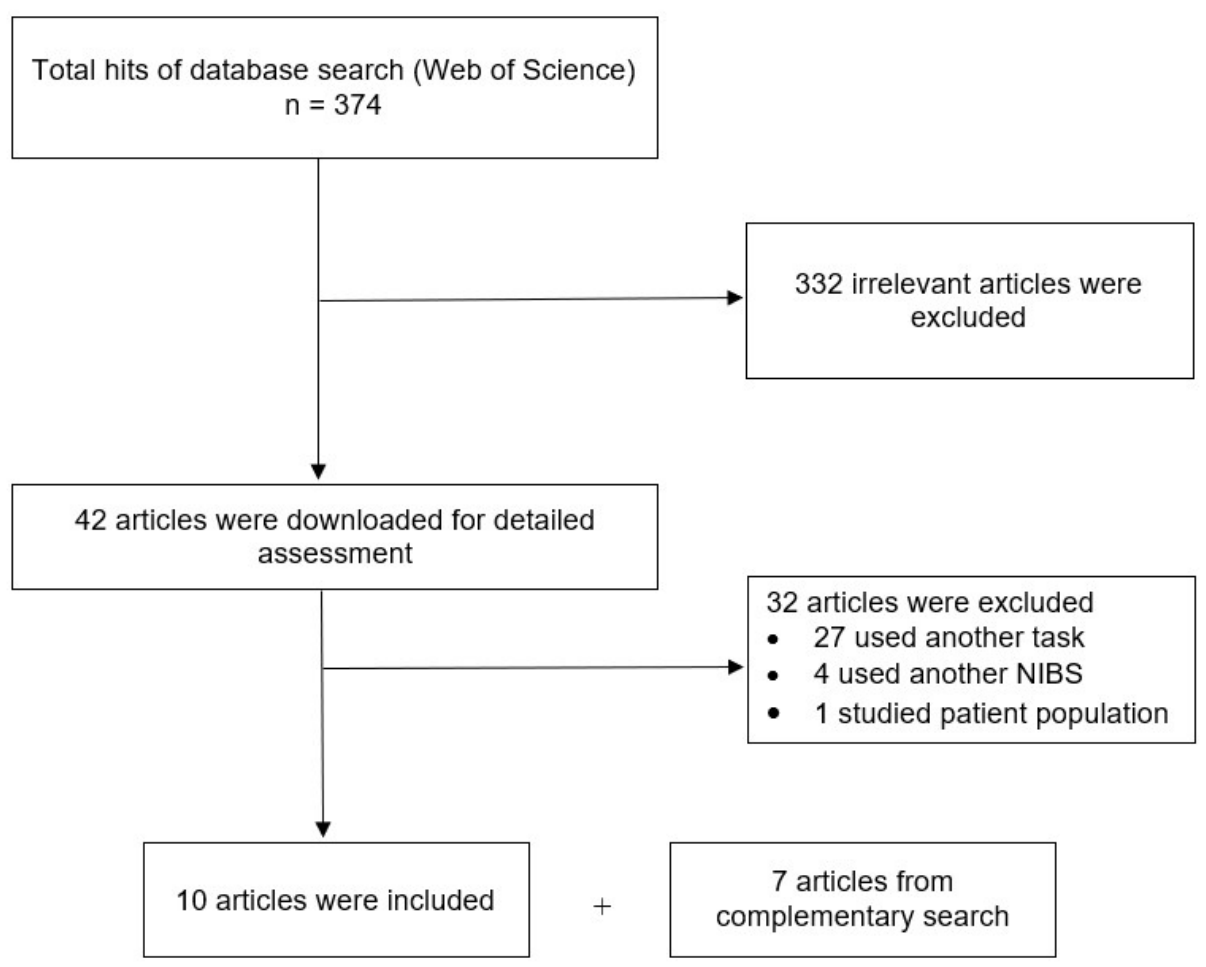

Figure 2. The flowchart of the literature search. Abbreviations. NIBS: non-invasive brain stimulation.

\section{What factors determine the effect of rTMS on sequence learning?}

Because the methodology of both SRTT and rTMS is highly diverse, we critically evaluated the studies based on the following parameters: (1) stimulated brain areas, (2) rTMS protocols (e.g., 'inhibitory' or 'facilitatory'), (3) stimulated hemisphere, (4) timing of the stimulation, (5) SRTT sequence properties, and (6) other methodological features (e.g., study design).

\section{Stimulated brain areas}

First, we examined the stimulated brain areas and found that the two most popular targets were the M1 (nine out of 17 studies) and the DLPFC (eight out of 17 studies). In addition, three studies targeted the SMA and four the parietal cortex. One study stimulated the Broca's area, and another one the cerebellum (see Table 1). Below, we evaluate the behavioral effects of rTMS in each cortical target separately. 
M1. The popularity of M1 as a stimulation target is attributed to its implicated role in the initial encoding of sequences and the early consolidation of already learned sequences (Muellbacher et al., 2002; Seidler et al., 2005). In the reviewed rTMS studies, typically, low-frequency rTMS or cTBS were applied over the M1, resulting in the weakening of the learning process (Clark et al., 2019; Experiment 1 in Rosenthal et al., 2009; Steel et al., 2016; Wilkinson et al., 2015), or the prevention of offline improvements (Breton \& Robertson, 2017; Robertson et al., 2005). Interestingly, two studies have found an increase in the visuomotor skill following lowfrequency rTMS or cTBS over M1. In one of them, low-frequency rTMS affected SRTT indirectly through the prevention of interference with a declarative task (Experiment 2 in Cohen \& Robertson, 2011). In the other study, cTBS abolished the decrease in corticospinal excitability, which allowed for offline improvements on an explicit SRTT (Experiment 3 in Tunovic et al., 2014). Only one of the nine studies attempted to use iTBS over M1, but it did not find any effect on implicit sequence learning (Wilkinson et al., 2010).

DLPFC. DLPFC has traditionally been identified as a brain area supporting executive functions and working memory (Miller \& Cohen, 2001; Yuan \& Raz, 2014). Lesion studies - where patients with prefrontal lesions show decreased sequence learning on the SRTT (Beldarrain et al., 2002; Beldarrain et al., 1999) - highlight its importance in visuomotor sequence learning, too. However, its functional role is still controversial (Janacsek \& Nemeth, 2013, 2015). Based on recent models, DLPFC may act as a neural switch between competitive memory processes (Ambrus et al., 2020; Daw et al., 2005; Wan Lee et al., 2014). On the one hand, it may favor declarative learning and memory (e.g., memory for events and facts), as well as top-down processes. Yet, if the situation requires acquiring new regularities (e.g., a completely new pattern or sequence), it recedes. Out of the eight studies, three studies found that rTMS over the DLPFC weakened implicit sequence learning (Experiment 2 in Cohen \& Robertson, 2011; Pascual-Leone et al., 1996; Robertson et al., 2001). In one of them, DLPFC stimulation reduced 
learning on the SRTT indirectly by interfering with a declarative task (Experiment 2 in Cohen \& Robertson, 2011). Examining explicit sequence learning, two of the six studies have found an enhancement for cTBS over the DLPFC (Galea et al., 2010; Experiment 2 in Tunovic et al., 2014). However, one recent study has found no effect of DLPFC stimulation on explicit SRTT (Gann et al., 2021). Two additional studies used probabilistic instead of deterministic sequences. One of them found that low-frequency rTMS over the DLPFC leads to better performance on this sequence type (also known as statistical learning) (Ambrus et al., 2020), while the other study found no effect of DLPFC stimulation on the performance (Wilkinson et al., 2010).

SMA. Some neuroimaging studies revealed that besides M1, another motor area, the SMA also appears to be involved during the SRTT (Hazeltine et al., 1997; Seidler et al., 2005). We found that only three studies have targeted the SMA. Two of them have found no association between SMA stimulation and learning on the SRTT (Pascual-Leone et al., 1996; Wilkinson et al., 2010). The third study has supported the role of the SMA in the intermanual transfer of the sequence (Perez et al., 2008), which refers to the phenomenon when the procedural knowledge acquired by one hand can be performed by the other hand (Grafton et al., 2002; Japikse et al., 2003). Due to the deficient number of publications, the functional role of the SMA in sequence learning remains elusive (Pascual-Leone et al., 1996; Wilkinson et al., 2010).

Parietal cortex. According to previous behavioral studies, a motor response is not strictly necessary for acquiring complex sequences; monitoring in itself can lead to learning (Nemeth et al., 2009; Song et al., 2008). Based on functional neuroimaging studies, the inferior parietal lobule (IPL) encodes the sequence at a general, abstract level, independently of the response mode (Grafton et al., 1998; Hikosaka et al., 1999). We found four studies that aimed to investigate the effect of rTMS over the parietal cortex on sequence learning. One study verified a crucial contribution of this area to perceptual sequence learning because the application of 
cTBS over the IPL resulted in the prevention of learning on a probabilistic SRTT (Experiment 2 in Rosenthal et al., 2009). According to the work of Breton and Robertson (2017), IPL seems to play a significant role in the consolidation of sequence knowledge as well, because lowfrequency rTMS blocked offline improvements on an implicit SRTT (Breton \& Robertson, 2017). In contrast, two of the four reviewed studies found no effect of stimulation of the IPL on sequence learning; therefore, its role remains elusive.

Broca's area. In the field of sequence learning, the Broca's area has been primarily tested on artificial grammar learning tasks (De Vries et al., 2010; Uddén et al., 2017), where participants need to extract rules from artificially generated grammatical sequences (Reber, 1967, 1989). Because the acquisition of the grammar of a language is connected to sequence learning (Nemeth et al., 2011), it is an interesting question whether this brain area is also involved in the acquisition of non-linguistic visuomotor sequences. Only one study has examined the role of Broca's area and showed that cTBS over the BA 44 prevented the learning on an implicit SRTT (Clerget et al., 2012).

Cerebellum. The role of the cerebellum in sequence learning is highly uncertain in the literature (Baetens et al., 2020; Janacsek et al., 2020). While the detrimental effect of cerebellar damage on sequence learning assumes its essential role (Dirnberger et al., 2013; Doyon et al., 1997; Gómez-Beldarrain et al., 1998; Shin \& Ivry, 2003), neuroimaging studies do not always support this hypothesis (Janacsek et al., 2020; Seidler et al., 2002; van der Graaf et al., 2006). So far, only one study investigated the causal role of the cerebellum and showed that low-frequency rTMS over the lateral cerebellum resulted in a significant weakening in sequence learning (Torriero et al., 2004). 


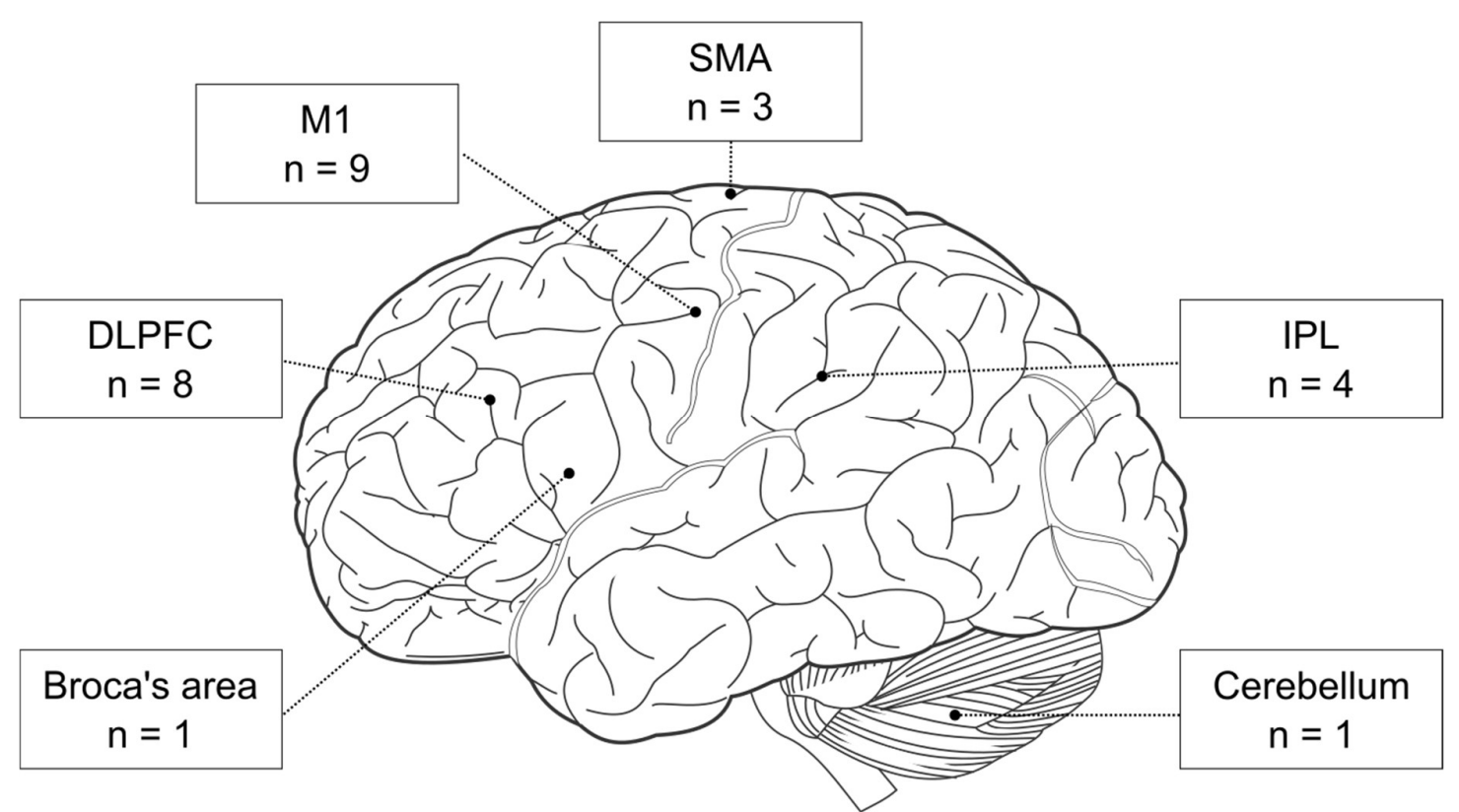

Figure 3. The cortical targets of rTMS-SRTT studies. Brain image was adapted from Hugh Guiney (https://commons.wikimedia.org/wiki/File:Human-brain.SVG; CC BY-SA 3.0).

\section{2. 'Inhibitory' and 'facilitatory' rTMS protocols}

It is common to assume a linear relationship between the direction of the produced aftereffects on cortical excitability and the behavioral effects of rTMS. According to this view, lowfrequency rTMS and cTBS (i.e., the 'inhibitory' protocols) might induce functional inhibition/disruption, whereas high-frequency rTMS and iTBS (i.e., the 'excitatory' protocols) might lead to functional improvements/enhancement. Although this generally accepted dichotomy between the stimulation frequency and the direction of the produced cognitive aftereffects is likely oversimplified, several studies discuss the results in this framework.

In the following, we delineate several reasons why it is challenging to predict the functional aftereffects of rTMS solely based on the protocol type (i.e., 'inhibitory' or 'excitatory'). First, there is a substantial interindividual variability when inducing corticospinal excitability changes in the M1. While group-level data might show frequency-dependent modulatory effects, they can vary significantly across individuals (Hamada et al., 2013; Maeda et al., 2000), and even within individuals (Goldsworthy et al., 2021). Second, it is unclear 
whether a given protocol that may decrease the cortical excitability in the M1 produces the same physiological effects in other cortical areas. For instance, some authors speculate that there might be overlaps in the produced aftereffects at least within the frontal cortex (e.g., M1 and DLPFC, as discussed in de Jesus et al., 2014). Third, this view may miss the brain's endogenous and dynamic compensatory mechanisms to external perturbations. For example, due to the interhemispheric compensation, decreasing the excitability level of the left DLPFC with low-frequency rTMS may lead to the compensatory recruitment of the right DLPFC (Ambrus et al., 2020). Fourth, the stimulation frequency is only one of many crucial stimulation parameters that can shape the direction of aftereffects. For example, the facilitating effect of high-frequency rTMS requires inter-train intervals; otherwise, it is more likely to produce an inhibitory effect (Rothkegel et al., 2010).

Based on these arguments, it is conceivable to expect that the behavioral effects of rTMS may not always match the alterations in cortical excitability. Consequently, 'facilitatory' protocols may not always enhance, and 'inhibitory' protocols may not necessarily weaken the performance. However, due to the prevailing use of these terms in the literature, we tentatively evaluated the link between the protocol type and the direction of the induced behavioral effects.

In the present review, 16 out of the 17 articles found rTMS effect on sequence learning, of which 12 studies followed the pattern that is intuitively expected based on the 'inhibitoryfacilitatory' dichotomy. These studies found that 'inhibitory' protocols (i.e., low-frequency rTMS, cTBS) indeed weakened the performance on the SRTT. However, three studies found that the 'inhibitory' stimulation of the DLPFC has led to enhanced performance on SRTT (Ambrus et al., 2020; Galea et al., 2010; Experiment 2 in Tunovic et al., 2014). These findings may be explained by the mediating role of the DLPFC between competitive memory processes (Ambrus et al., 2020; Daw et al., 2005). Alternatively, the behavioral effects of rTMS may be due to changes in the cortical excitability of another brain area that is, however, part of the same 
neural network. For example, Cao and his colleagues (2018) found that 'facilitatory' iTBS over the DLPFC decreased, while 'inhibitory' cTBS over the DLPFC increased the cortical excitability in the M1 (Cao et al., 2018). In another study, rTMS affected SRTT performance indirectly by preventing interference with a declarative task (Experiment 2 in Cohen \& Robertson, 2011).

Out of the 17 reviewed studies, only three applied 'facilitatory' protocols, and only one revealed its effect on sequence learning (Pascual-Leone et al., 1996). This study showed that 5 Hz rTMS (Pascual-Leone et al., 1996) weakened the performance on the SRTT. However, the other two studies found no effects of rTMS (Gann et al., 2021; Wilkinson et al., 2010). Therefore, the impact of 'facilitatory' protocols ( $\geq 5 \mathrm{~Hz}$ and iTBS) on SRTT is still lacking and requires further exploration. 


\begin{tabular}{|c|c|c|c|c|c|c|}
\hline Target area & Authors and year & $\begin{array}{l}\text { rTMS } \\
\text { protocol }\end{array}$ & Hemisphere & $\begin{array}{c}\text { Timing of } \\
\text { stimulation }\end{array}$ & Type of sequence & Outcome \\
\hline \multicolumn{7}{|l|}{ M1 } \\
\hline & Robertson et al., 2005 & $1 \mathrm{~Hz}$ rTMS & Left only & After learning & 12-item implicit deterministic & Blocked offline improvements over the day, but not overnight \\
\hline & Breton \& Robertson, 2017 & $1 \mathrm{~Hz}$ rTMS & Left only & After learning & $\begin{array}{l}\text { 12-item implicit/explicit } \\
\text { deterministic }\end{array}$ & Blocked offline improvements in explicit, but not in implicit task \\
\hline & $\begin{array}{l}\text { Tunovic et al., } 2014 \\
\text { (Experiment } 3 \text { ) }\end{array}$ & cTBS & Right only & After learning & 12-item explicit deterministic & Offline improvements after cTBS \\
\hline & $\begin{array}{l}\text { Cohen \& Robertson, } 2011 \\
\text { (Experiment 2) }\end{array}$ & $1 \mathrm{~Hz}$ rTMS & Right only & After learning & 12-item implicit deterministic & $\begin{array}{l}\text { Increased learning after } 12 \mathrm{~h} \text { consolidation by preventing interference } \\
\text { with a declarative task }\end{array}$ \\
\hline & Wilkinson et al., 2010 & cTBS, iTBS & Left only & Before learning & 12-item implicit probabilistic & Learning was prevented by cTBS \\
\hline & Wilkinson et al., 2015 & cTBS & Left only & Before learning & 12-item implicit probabilistic & Decreased initial sequence learning and recall \\
\hline & Steel et al., 2016 & cTBS & Left only & Before learning & 12-item implicit probabilistic & Learning was disrupted \\
\hline & $\begin{array}{l}\text { Rosenthal et al., } 2009 \\
\text { (Experiment 1,2) }\end{array}$ & cTBS & Contralateral to dominant hand & Before learning & 12-item implicit probabilistic & Learning was disrupted in manual, but not in perceptual task \\
\hline & Clark et al., 2019 & cTBS & Left only & Before learning & 12-item implicit deterministic & Decreased learning in simple, but not in a more complex sequence \\
\hline \multicolumn{7}{|l|}{ DLPFC } \\
\hline & Pascual-Leone et al., 1996 & $5 \mathrm{~Hz}$ rTMS & $\begin{array}{l}\text { Left or right in separate } \\
\text { conditions }\end{array}$ & During learning & 12-item implicit deterministic & Learning was disrupted \\
\hline & Robertson et al., 2001 & $1 \mathrm{~Hz}$ rTMS & Contralateral to dominant hand & Before learning & 10-item implicit deterministic & Learning was prevented in spatial, but not in color cue guided task \\
\hline & Gann et al., 2021 & cTBS, iTBS & Left only & Before learning & 8 -item explicit deterministic & No effect on learning \\
\hline & Galea et al., 2010 & cTBS & Left or right in separate groups & After learning & 12-item explicit deterministic & Improved learning after $8 \mathrm{~h}$ consolidation \\
\hline & $\begin{array}{l}\text { Tunovic et al., } 2014 \\
\text { (Experiment 2) }\end{array}$ & cTBS & Right only & After learning & 12-item explicit deterministic & Offline improvements after cTBS \\
\hline & $\begin{array}{l}\text { Cohen \& Robertson, } 2011 \\
\text { (Experiment 2) }\end{array}$ & $1 \mathrm{~Hz}$ rTMS & Right only & After learning & 12-item implicit deterministic & $\begin{array}{l}\text { Decreased learning after } 12 \mathrm{~h} \text { consolidation by fail to prevent } \\
\text { interference with a declarative task }\end{array}$ \\
\hline & Ambrus et al., 2020 & $1 \mathrm{~Hz}$ rTMS & Bilaterally & $\begin{array}{l}\text { Between learning } \\
\text { blocks }\end{array}$ & 8-item implicit probabilistic & Improved learning after $24 \mathrm{~h}$ consolidation \\
\hline \multicolumn{7}{|l|}{ Broca's area } \\
\hline & Clerget et al., 2012 & cTBS & Left only & Before learning & 20-item implicit deterministic & Learning was prevented \\
\hline \multicolumn{7}{|l|}{ SMA } \\
\hline & Pascual-Leone et al., 1996 & $5 \mathrm{~Hz} \mathrm{rTMS}$ & not available & During learning & 12-item implicit deterministic & No effect on learning \\
\hline
\end{tabular}




\begin{tabular}{|c|c|c|c|c|c|}
\hline Wilkinson et al., 2010 & cTBS & Left only & Before learning & 12-item implicit probabilistic & No effect on learning \\
\hline Perez et al., 2008 & $1 \mathrm{~Hz}$ rTMS & Left only & During learning & 12-item implicit deterministic & Blocked intermanual transfer of the skill \\
\hline Robertson et al., 2001 & $1 \mathrm{~Hz}$ rTMS & $\begin{array}{c}\text { Contralateral to the dominant } \\
\text { hand }\end{array}$ & Before learning & 10-item implicit deterministic & No effect on learning \\
\hline $\begin{array}{l}\text { Rosenthal et al., } 2009 \\
\text { (Experiment 1,2) }\end{array}$ & cTBS & Right only & Before learning & 12-item implicit probabilistic & Learning was disrupted in perceptual, but not in manual task \\
\hline Breton \& Robertson, 2017 & $1 \mathrm{~Hz}$ rTMS & Left only & After learning & $\begin{array}{c}\text { 12-item implicit, explicit } \\
\text { deterministic }\end{array}$ & Blocked offline improvements in implicit, but not in explicit task \\
\hline Clark et al., 2019 & cTBS & Left only & Before learning & 12-item implicit, deterministic & No effect on learning \\
\hline
\end{tabular}

Cerebellum

Torriero et al., 2004

$1 \mathrm{~Hz}$ rTMS Left or right in separate groups

Before learning

12-item implicit, deterministic $\quad$ Learning was disrupted

Table 1. The effect of different rTMS protocols on visuomotor sequence learning according to the target area, sequence type, and timing of the stimulation 


\section{Stimulated hemisphere(s)}

Many studies used rTMS to better understand the hemispheric involvement of a given brain region when performing the SRTT. These studies typically ask whether the left or right brain area (e.g., M1) is causally involved in a specific task phase (e.g., learning phase). To this aim, most studies stimulated the left or the right hemisphere at a time and studied whether rTMS could modulate the performance.

Performing the SRTT may require using only one hand or both hands. When the participants perform the SRTT with only one hand (e.g., the right hand), the stimulation may target the contralateral (i.e., left) or ipsilateral (i.e., right) hemisphere. Eleven of the 17 studies targeted the contralateral hemisphere (see Table 1). In studies where only the left hemisphere was stimulated, only right-handed participants were included (see Table 1).

Yet, studies targeting the dominant hemisphere (based on the M1) may miss the possibility that the non-stimulated hemisphere can take over the function of the stimulated one (Andoh \& Martinot, 2008; Sack et al., 2005), potentially influencing the results. Applying sequential bilateral stimulations (i.e., consecutively delivering the same rTMS protocol over a given cortical target on both hemispheres) may be a promising solution to overcome the possible interhemispheric compensatory mechanisms (Ambrus et al., 2020).

Considering the side of the stimulation, some studies targeted both hemispheres in separate experimental groups. Using this method, Galea and her colleagues (2010) successfully demonstrated that cTBS over the right DLPFC improved visuomotor sequence learning to a greater extent than the left DLPFC (Galea et al., 2010). Another study revealed a dissociation between cerebellar hemispheres: the stimulation of the right cerebellar hemisphere weakened sequence learning regardless of which hand was used, while the interference with the left cerebellar hemisphere affected only through the ipsilateral hand (Torriero et al., 2004). Therefore, this method is suitable for exploring potential lateralization effects as well. 
For non-motor brain areas, it is worth targeting both hemispheres separately and applying bilateral protocols. This approach can avoid hemispheric compensatory mechanisms and reveal possible dissociation between hemispheres (see Ambrus et al., 2020). Furthermore, the sequential bilateral stimulation for the two-handed version of the task may be a particularly good solution.

\section{Timing of the stimulation}

Sequence learning is a multi-stage process: it consists of the learning, consolidation, and recall phases. Slightly different brain regions and neural networks may be recruited at each learning stage (Veldman et al., 2018); therefore, we evaluated the studies in each stage separately.

Learning phase. One can deliver rTMS immediately before or during the learning phase (see Figure 4). During the learning phase, rTMS may be applied simultaneously with the task performance or between the learning blocks. Only two studies have applied rTMS during the initial sequence learning process (so-called „online stimulation”). In one study, high-frequency rTMS over the DLPFC led to a performance decrease on the SRTT (Pascual-Leone et al., 1996). In a more recent study, the authors found performance improvement on an alternating SRTT when stimulating the DLPFC with low-frequency rTMS (Ambrus et al., 2020). Most research delivered stimulation immediately before task performance (so-called „offline stimulation”; see Table 1).

Consolidation phase. We may apply rTMS after the learning phase to verify its effect on memory consolidation (see Figure 4). In one study, cTBS over the DLPFC improved performance after an 8-hour-long offline period (Galea et al., 2010). In contrast, low-frequency rTMS over the M1 blocked offline improvements on an implicit SRTT over the day (Robertson et al., 2005), as well as on an explicit SRTT after sleep (Breton \& Robertson, 2017). We conclude that rTMS over the DLPFC and M1 can influence the development of memory traces because their stimulation leads to changes in the consolidation process. 
Recall phase. Finally, rTMS may be applied immediately before or during the recall phase. However, to the best of our knowledge, no studies have applied rTMS immediately before or during the recall phase to date. Thus, it is unclear whether rTMS can modulate the recall of a well-acquired sequence knowledge.

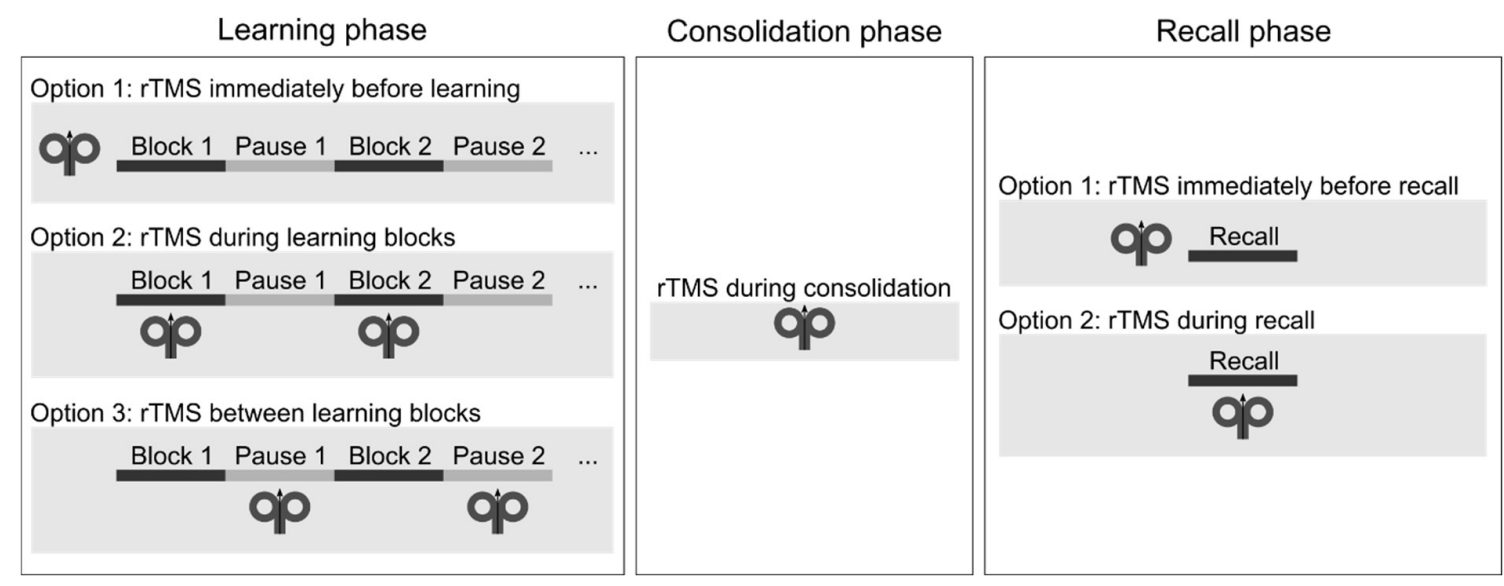

Figure 4. Timing of the stimulation in each task phase.

\section{Type of the SRTT sequence}

The effect of stimulation may depend on the SRTT sequence type. Several new versions of the SRTT have emerged that can differ in three crucial dimensions: the sequence applied can be (1) implicit or explicit, (2) deterministic or probabilistic, and (3) first-order conditional (FOC) or second-order conditional (SOC) (as defined in section Different variations of the SRTT).

Implicit vs. explicit sequences. The most commonly used version of SRTT uses implicit sequences (here, 13 out of the 17 reviewed studies used the implicit SRTT). However, its explicit version (i.e., the existence of the predetermined sequence is revealed to the participants before learning) can also be applied if the goal is to test intentional learning or declarative knowledge of the sequence. Among the reviewed rTMS articles, we found that rTMS could affect explicit and implicit learning (Table 1). However, the study of Breton and Robertson (2017) showed evidence that the degree of sequence awareness can influence the effect of 
rTMS. They examined the role of M1 and IPL in both implicit and explicit SRTT and revealed a double dissociation: low-frequency rTMS over the IPL prevented offline improvement in the implicit, but not in the explicit task. On the other hand, the same stimulation over the M1 prevented offline improvement in the explicit but not in the implicit task (Breton \& Robertson, 2017).

Deterministic vs. probabilistic sequences. In the classical SRTT, stimuli follow a fixed order, creating a deterministic sequence (Nissen \& Bullemer, 1987; Shanks, 2005). Probabilistic types of SRTT also exist, where the sequence is hidden in noise; therefore, learning is more likely to remain implicit (Howard et al., 2004; Song et al., 2007). Five out of 17 studies used probabilistic sequences, and 12 used deterministic sequences (Table 1). All of the studies employing probabilistic sequence learning tasks found behavioral effects of rTMS. In four studies, the learning deteriorated (Experiment 1 and 2 in Rosenthal et al., 2009; Steel et al., 2016; Wilkinson et al., 2010, 2015), and in one study, learning was improved (Ambrus et al., 2020). On the other hand, deterministic sequence learning performance was successfully manipulated in 11 out of the 12 studies: in eight studies, learning was disrupted (Breton \& Robertson, 2017; Clark et al., 2019; Clerget et al., 2012; Pascual-Leone et al., 1996; Perez et al., 2008; Robertson et al., 2005, 2001; Torriero et al., 2004), and in three studies learning was improved (Experiment 2 in Cohen \& Robertson, 2011; Galea et al., 2010; Experiment 2 and 3 in Tunovic et al., 2014). Therefore, it seems that rTMS can equally modify the learning of both probabilistic and deterministic sequences. However, we cannot draw firm conclusions due to the low number of studies with probabilistic sequences.

FOC vs. SOC sequences. Another critical factor is the statistical structure of the sequence. In the simpler first-order conditional (FOC) sequences, elements can be predicted by the preceding one. On the other hand, in the more complex second-order conditional (SOC) sequences, it is the combination of two consecutive elements that predicts the forthcoming one. Clark and his 
colleagues (2019) investigated the role of M1 in the acquisition of simpler FOC and more complex SOC sequences. According to their findings, cTBS over the M1 resulted in poorer learning of the FOC sequence compared to the SOC sequence. These findings support the hypothesis that the acquisition of FOC and SOC sequences may rely on different neural networks: simpler FOC sequences are processed by a circuitry involving the M1, while more complex SOC sequences are associated with an expanded network, including Brodmann area 44 (BA44) and DLPFC (Ashe et al., 2006; Lum et al., 2018). Based on these promising results, future studies may investigate FOC and SOC sequences targeting non-motor areas too.

\section{Other methodological features}

Next, we summarize the methodological aspects of rTMS studies, focusing on the research design, the type of control, the localization techniques of target areas, the determination of the stimulation intensity, and the interpretation of different outcome measures (see Table 2 and Table 3).

Table 2. Research design and power

\begin{tabular}{|c|c|c|c|c|}
\hline Design & Study & Type of control condition & $\mathbf{N}$ & n/group \\
\hline \multicolumn{5}{|c|}{ Within-subjects } \\
\hline & Pascual-Leone et al., 1996 & Absence of stimulation & 7 & \\
\hline & Robertson et al., 2001 & Active control (parietal cortex) & 6 & \\
\hline & Gann et al., 2021 & No control & 19 & \\
\hline & Steel et al., 2016 & Sham stimulation with placebo coil & 22 & \\
\hline \multicolumn{5}{|c|}{ Between-subjects } \\
\hline & Robertson et al., 2005 & Sham stimulation with placebo coil, time and site control & 36 & 6 \\
\hline & Breton \& Robertson, 2017 & Sham stimulation with placebo coil & 67 & $12-16$ \\
\hline & $\begin{array}{l}\text { Tunovic et al., } 2014 \\
\text { (Experiment 2) }\end{array}$ & Intermediate TBS, localization control & 24 & 12 \\
\hline & $\begin{array}{l}\text { Tunovic et al., } 2014 \\
\text { (Experiment 3) }\end{array}$ & Intermediate TBS & 24 & 12 \\
\hline & $\begin{array}{l}\text { Cohen \& Robertson, } 2011 \\
\text { (Experiment 2) }\end{array}$ & Sham stimulation over vertex with placebo coil & 40 & 10 \\
\hline & Wilkinson et al., 2010 & Sham stimulation with $90^{\circ}$ rotation of the coil & 40 & 8 \\
\hline & $\begin{array}{l}\text { Rosenthal et al., } 2009 \\
\text { (Experiment 1,2) }\end{array}$ & Sham stimulation over vertex with placebo coil & 24,24 & 8,8 \\
\hline & Galea et al., 2010 & Active control (occipital cortex) & 30 & 10 \\
\hline & Ambrus et al., 2020 & Sham stimulation with $90^{\circ}$ rotation of the coil & 31 & 15 and 16 \\
\hline
\end{tabular}




$\begin{array}{lccc}\text { Clerget et al., } 2012 & \text { Active control (vertex) } & 17 & 8 \text { and } 9 \\ \text { Perez et al., 2008 } & \text { Sham stimulation with a second coil discharged in the air } & 33 & 11 \\ \text { Torriero et al., 2004 } & \text { Absence of stimulation } & 36 & 5-7\end{array}$

Mixed design

Wilkinson et al., 2015

Sham stimulation with placebo coil

$40 \quad 20$

Clark et al., 2019

Sham stimulation with placebo coil

$48 \quad 16$

Research design and the type of control condition. The reviewed rTMS studies mostly applied between-subjects research designs, and it was less common to use within-subjects or mixed study designs (see Table 2). Typically, the stimulation group is compared to a control group that goes through the same procedure except for the stimulation parameters. A frequent study design is when the control group receives sham stimulation (i.e., using a placebo coil or tilting the coil away from the skull to a certain degree). One known limitation of this approach is that the rTMS-induced perceptual adverse effects (e.g., somatosensory sensations - in part — due to cranial muscle contractions) are not fully comparable to the verum stimulation. Therefore, the blinding of the participants might not be maintained entirely (Duecker \& Sack, 2015).

Instead of the sham condition, studies may apply active control condition. Here, the stimulation is delivered over a 'control' site while using the identical stimulation protocol as for the main cortical. This option has the disadvantage that the brain area chosen as the control site might play a role in the tested function because the underlying brain networks involved in the given task might not be fully revealed yet.

In the reviewed studies, sham stimulation was the most commonly chosen approach here, ten studies used it as a control. On the other hand, three studies chose active control with different control sites (i.e., vertex, occipital and parietal sites). In two studies, members of the control group were simply not exposed to rTMS (Pascual-Leone et al., 1996; Torriero et al., 2004), one study compared cTBS to intermediate TBS (Experiment 2 and 3 in Tunovic et al., 2014) and another study only compared two active stimulation protocols (cTBS and iTBS) to each other (Gann et al., 2021) (see Table 2). 
Localization technique. The different methods of target area localization may also contribute to the inconsistency of results (De Witte et al., 2018; Sack et al., 2009). The target area for M1 is traditionally identified during the motor threshold measurement (see Table 3). In the reviewed studies, non-motor cortical regions were typically located by MRI-guided neuronavigation systems using individual MRIs. Most of them successfully demonstrated the causal role of the given regions of the sequence learning process (Ambrus et al., 2020; Clerget et al., 2012; Galea et al., 2010; Experiment 1 and 2 in Rosenthal et al., 2009). Wilkinson and her colleagues (2010) localized their non-motor target areas by the 10-20 EEG system or by the 5-cm rule with M1 as a reference. Interestingly, they did not find any effect (Wilkinson et al., 2010). As DLPFC is an extensive area, different localization techniques lead to slightly different regions (e.g., BA9, BA46). According to an fMRI investigation, BA9 shows greater activity during sequence learning than BA46 in young adults (Aizenstein et al., 2006). Accordingly, localization may be an important parameter, and future studies should preferably use neuronavigation to precisely target a given cortical region.

Stimulation intensity and the total number of pulses. The overwhelming majority of rTMS studies determine the stimulation intensity based on the motor threshold (Turi et al., 2021). In line with this, 16 of the 17 studies used the motor threshold; however, the parameters of the intensity selection ranged substantially (see Table 3 ). Only one study used a fixed intensity (Ambrus et al., 2020); however, it successfully modulated the SRTT performance. Although adjusting the intensity to the motor threshold is a reasonable method for M1 stimulation, the physiological justification for this procedure is less clear for non-motor cortical areas. Besides, the high diversity of the chosen intensities might make it even harder to compare the results of different studies. 
In addition to the stimulation intensity, the total number of pulses can also be decisive for the effectiveness of rTMS (Thut \& Pascual-Leone, 2010). Specific stimulation protocols may standardize the total number of pulses. In 12 out of the 17 reviewed articles, the number of the delivered pulses was 600 . Three studies used more (i.e., 900, 1200, and 300), whereas one study used fewer pulses (i.e., 300; see Table 3). Even with higher or lower delivered pulses, a significant effect of stimulation was demonstrated. 
Table 3. Methodological details of the studies

\begin{tabular}{|c|c|c|c|c|}
\hline Study & Localization technique & Intensity & $\begin{array}{l}\text { Total number of } \\
\text { pulses }\end{array}$ & Data type of the result \\
\hline $\begin{array}{l}\text { Pascual-Leone et al., } \\
1996\end{array}$ & Contractions in anterior tibialis or the abductor pollicis muscles & $115 \%$ of MT & not available & $\mathrm{RT}$ \\
\hline Robertson et al., 2001 & Contractions in abductor pollicis brevis muscle, 10-20 EEG system & $115 \%$ of MT & 900 & RT \\
\hline Robertson et al., 2005 & Contractions in abductor pollicis brevis muscle & $90 \%$ of MT & 600 & Learning score based on RT \\
\hline $\begin{array}{l}\text { Breton \& Robertson, } \\
2017\end{array}$ & $\begin{array}{l}\text { Contractions in abductor pollicis brevis muscle, MRI-guided } \\
\text { neuronavigation }\end{array}$ & $90 \%$ of MT & 600 & Learning improvement based on RT \\
\hline $\begin{array}{l}\text { Tunovic et al., } 2014 \\
\text { (Experiment } 2 \text { and } 3 \text { ) }\end{array}$ & MRI-guided neuronavigation, optimal location for inducing MEPs & $80 \%$ of AMT & 600 & Learning score based on RT \\
\hline $\begin{array}{l}\text { Cohen \& Robertson, } 2011 \\
\text { (Experiment 2) }\end{array}$ & $\begin{array}{l}\text { MRI-guided neuronavigation, contractions in abductor pollicis brevis } \\
\text { muscle }\end{array}$ & $90 \%$ of MT & 600 & Learning score based on RT \\
\hline $\begin{array}{l}\text { Rosenthal et al., } 2009 \\
\text { (Experiment 1,2) }\end{array}$ & MRI-guided neuronavigation & $70 \%$ of RMT & 300 & Learning score based on RT \\
\hline Wilkinson et al., 2010 & Location of hand representation, 10-20 EEG system & $80 \%$ of AMT & 600 & Learning score based on RT \\
\hline Wilkinson et al., 2015 & Location of hand representation, MRI-guided neuronavigation & $80 \%$ of AMT & 600 & $\begin{array}{l}\text { Learning score based on RT and } \\
\text { accuracy }\end{array}$ \\
\hline Steel et al., 2016 & Location of hand representation, MRI-guided neuronavigation & $80 \%$ of AMT & 600 & Learning score based on RT \\
\hline Torriero et al., 2004 & $1 \mathrm{~cm}$ under and $3 \mathrm{~cm}$ left/right to the inion & $90 \%$ of MT & 600 & RT \\
\hline Perez et al., 2008 & Relative to the activation of the left tibialis anterior muscle & $80 \%$ of RMT & 1200 & RT \\
\hline Galea et al., 2010 & MRI-guided neuronavigation & $80 \%$ of AMT & 600 & Learning improvement based on RT \\
\hline Clerget et al., 2012 & MRI-guided neuronavigation & $80 \%$ of RMT & 600 & RT \\
\hline Clark et al., 2019 & RMT, MRI-guided neuronavigation & $70 \%$ of RMT & 600 & RT \\
\hline Gann et al., 2021 & MRI-guided neuronavigation & $80 \%$ of AMT & 600 & RT and accuracy \\
\hline Ambrus et al., 2020 & MRI-guided neuronavigation & $55 \%$ of MSO & $\begin{array}{c}3000 \\
(1500 / \text { hemisphere })\end{array}$ & Learning score based on RT \\
\hline
\end{tabular}




\section{Discussion}

In this review, we examined the most critical factors that govern the behavioral effects of rTMS on visuomotor sequence learning as measured by the SRTT. We critically explored the role of rTMS parameters (e.g., frequency, timing, etc.) and the task characteristics (e.g., sequence type). Moreover, we scrutinized the reviewed studies based on additional methodological details (e.g., study design, localization technique).

We found that the two most frequently stimulated cortical targets were the M1 and DLPFC, and only a few studies have targeted the parietal, supplemental motor, and cerebellar areas. While low-frequency rTMS and cTBS (i.e., 'inhibitory' protocols) over the M1 weakened sequence learning, the results are less consistent for the DLPFC. In some studies, low-frequency rTMS and cTBS over the DLPFC weakened learning (Pascual-Leone et al., 1996; Robertson et al., 2001). However, more recent studies have found no effects (Gann et al., 2021; Wilkinson et al., 2010) or even enhanced learning (Ambrus et al., 2020; Galea et al., 2010). One possible explanation for the behavioral improvement might lie in the competitive relationship between the so-called more- and less-controlled learning processes (Daw et al., 2005). Given that the DLPFC might play a mediating role between these two learning processes, low-frequency rTMS may promote the less-controlled, associative learning processes via its implicated inhibitory effects (Ambrus et al., 2020; Daw et al., 2005). Taken together, our findings suggest that stimulating the M1 or the DLPFC could modulate sequence learning; however, the underlying mechanisms and the reasons behind the discrepancies between studies should be clarified in the future.

We found that most studies used 'inhibitory' rTMS protocols with the aim to reduce the cortical excitability level of the targeted brain areas and weaken sequence learning (Breton \& Robertson, 2017; Clark et al., 2019; Clerget et al., 2012; Perez et al., 2008; Experiment 1 and 2 in Rosenthal et al., 2009; Steel et al., 2016; Torriero et al., 2004; Wilkinson et al., 2015). In 
contrast, only a handful of studies used 'facilitatory' rTMS, and they mainly led to null findings (Gann et al., 2021; Wilkinson et al., 2010). One possible explanation for the observed pattern of findings is that it might be more challenging to enhance cognitive performance in healthy participants. Moreover, we should not rule out the potential file-drawer effect because substantially fewer studies published 'facilitatory' than 'inhibitory' protocols. Nevertheless, studies using high-frequency rTMS and iTBS are warranted to reveal the efficacy of these protocols to modify sequence learning.

Our findings also revealed that the stimulation parameters, the task characteristics, and the study designs were highly diverse in the reviewed studies, which could have influenced the results to a great extent. This diversity makes the direct comparisons between the studies challenging. Different stimulation protocols (i.e., conventional rTMS vs. TBS), stimulation timings (i.e., before, during, or after learning), control stimulation (i.e., no control, sham stimulation, active control), and stimulation intensities were utilized in almost all studies. Based on the protocol type, stimulation intensity, stimulation frequency, stimulation timing, the total number of pulses, and the control stimulation, 11 out of the 17 studies used unique protocols. In the remaining six studies, we found two stimulation protocols that were repeatedly used. We also noted that these two protocols belonged to two research groups. Thus, a total of 13 unique research protocols emerged out of the 17 studies. In addition to answering new questions and testing novel methods, it seems necessary to verify whether the already existing key findings are replicable with the same (i.e., direct replication) or slightly different stimulation parameters (i.e., conceptual replication).

Although both repetitive stimulation techniques seem to modulate visuomotor sequence learning effectively, a recent study suggests that the conventional low-frequency rTMS has a greater inhibitory effect on motor sequence learning than cTBS (Glinski, 2021). Therefore, 
future studies may explore the potential differences in the efficacy of rTMS and cTBS protocols (along with the different stimulation parameters) in modulating visuomotor sequence learning.

\section{Conclusion and outlook}

Here, we reviewed the most important factors that are worth considering when studying visuomotor sequence learning via rTMS. Based on the reviewed studies, we conclude that rTMS could produce exciting behavioral findings when targeting the M1 and DLPFC. Yet, we know little about the involvement of other cortical regions and neural networks that underlie visuomotor sequence learning. The cerebellar, parietal, and supplementary motor areas are promising cortical targets to modulate sequence learning, and hence, they deserve further investigations. Similarly, it may be worthwhile to create research series to examine the role of the relevant brain areas in all three learning phases (i.e., sequence acquisition, consolidation, and recall). Directly comparing the effects of rTMS on different sequence characteristics (e.g., FOC vs. SOC sequences, probabilistic vs. deterministic sequences) could also deepen our understanding of the brain mechanisms underlying visuomotor sequence learning. Future studies may combine rTMS with electrophysiological and/or neuroimaging methods to gain a clearer view of the underlying behavioral and neural effects of rTMS on visuomotor sequence learning.

\section{Acknowledgment}

The authors are thankful to Edwin Robertson for his comments on a previous version of the manuscript. This research was supported by the IDEXLYON Fellowship of the University of Lyon as part of the Programme Investissements d'Avenir (ANR-16-IDEX-0005) (to D.N.); National Brain Research Program (project 2017-1.2.1-NKP-2017-00002). Project no. 128016 has been implemented with the support provided by the Ministry of Innovation and Technology 
of Hungary from the National Research, Development and Innovation Fund, financed under the NKFI/OTKA K funding scheme (to D.N.). Prepared with the support of the Richter Gedeon Talentum Foundation established by Richter Gedeon Plc. (headquarters: 1103 Budapest, Gyömröi út 19-21.), in the framework of the "Richter Gedeon Excellence PhD Scholarship" (to L. Sz-B.). 


\section{References}

Aizenstein, H. J., Butters, M. A., Clark, K. A., Figurski, J. L., Andrew Stenger, V., Nebes, R. D., Reynolds, C. F., \& Carter, C. S. (2006). Prefrontal and striatal activation in elderly subjects during concurrent implicit and explicit sequence learning. Neurobiology of Aging. https://doi.org/10.1016/j.neurobiolaging.2005.03.017

Ambrus, G. G., Vékony, T., Janacsek, K., Trimborn, A. B. C., Kovács, G., \& Nemeth, D. (2020). When less is more: Enhanced statistical learning of non-adjacent dependencies after disruption of bilateral DLPFC. Journal of Memory and Language. https://doi.org/10.1016/j.jml.2020.104144

Andoh, J., \& Martinot, J. L. (2008). Interhemispheric compensation: A hypothesis of TMSinduced effects on language-related areas. European Psychiatry. https://doi.org/10.1016/j.eurpsy.2007.10.012

Ashe, J., Lungu, O. V., Basford, A. T., \& Lu, X. (2006). Cortical control of motor sequences. In Current Opinion in Neurobiology. https://doi.org/10.1016/j.conb.2006.03.008

Baetens, K., Firouzi, M., Van Overwalle, F., \& Deroost, N. (2020). Involvement of the cerebellum in the serial reaction time task (SRT) (Response to Janacsek et al.). In NeuroImage. https://doi.org/10.1016/j.neuroimage.2020.117114

Bagnato, S., Currà, A., Modugno, N., Gilio, F., Quartarone, A., Rizzo, V., Girlanda, P., Inghilleri, M., \& Berardelli, A. (2005). One-hertz subthreshold rTMS increases the threshold for evoking inhibition in the human motor cortex. Experimental Brain Research. https://doi.org/10.1007/s00221-004-2020-0

Beldarrain, M. G., Grafman, J., Pascual-Leone, A., \& Garcia-Monco, J. C. (1999). Procedural learning is impaired in patients with prefrontal lesions. Neurology. https://doi.org/10.1212/wnl.52.9.1853

Beldarrain, M., Gafman, J., Ruiz de Velasco, I., Pascual-Leone, A., \& Garcia-Monco, J. (2002). Prefrontal lesions impair the implicit and explicit learning of sequences on visuomotor tasks. Experimental Brain Research. https://doi.org/10.1007/s00221-001-0935-2

Bergmann, T. O., \& Hartwigsen, G. (2020). Inferring causality from noninvasive brain stimulation in cognitive neuroscience. Journal of Cognitive Neuroscience. https://doi.org/10.1162/jocn_a_01591

Bergstrom, J. C., Howard, J. H., \& Howard, D. V. (2012). Enhanced Implicit Sequence Learning in College-age Video Game Players and Musicians. Applied Cognitive Psychology. https://doi.org/10.1002/acp.1800 
Breton, J., \& Robertson, E. M. (2017). Dual enhancement mechanisms for overnight motor memory consolidation. Nature Human Behaviour. https://doi.org/10.1038/s41562-0170111

Cao, N., Pi, Y., Liu, K., Meng, H., Wang, Y., Zhang, J., Wu, Y., \& Tan, X. (2018). Inhibitory and facilitatory connections from dorsolateral prefrontal to primary motor cortex in healthy humans at rest-An rTMS study. Neuroscience Letters. https://doi.org/10.1016/j.neulet.2018.09.032

Cárdenas-Morales, L., Nowak, D. A., Kammer, T., Wolf, R. C., \& Schönfeldt-Lecuona, C. (2010). Mechanisms and applications of theta-burst rTMS on the human motor cortex. In Brain Topography. https://doi.org/10.1007/s10548-009-0084-7

Chouinard, P. A., Leonard, G., \& Paus, T. (2005). Role of the primary motor and dorsal premotor cortices in the anticipation of forces during object lifting. Journal of Neuroscience. https://doi.org/10.1523/JNEUROSCI.4649-04.2005

Clark, G. M., Barham, M. P., Ware, A. T., Plumridge, J. M. A., O’Sullivan, B., Lyons, K., Fitzgibbon, T., Buck, B., Youssef, G. J., Ullman, M. T., Enticott, P. G., \& Lum, J. A. G. (2019). Dissociable implicit sequence learning mechanisms revealed by continuous thetaburst stimulation. Behavioral Neuroscience. https://doi.org/10.1037/bne0000299

Clerget, E., Poncin, W., Fadiga, L., \& Olivier, E. (2012). Role of Broca's area in implicit motor skill learning: Evidence from continuous theta-burst magnetic stimulation. Journal of Cognitive Neuroscience. https://doi.org/10.1162/jocn_a_00108

Cohen, D. A., \& Robertson, E. M. (2011). Preventing interference between different memory tasks. Nature Neuroscience. https://doi.org/10.1038/nn.2840

Daselaar, S. M., Rombouts, S. A. R. B., Veltman, D. J., Raaijmakers, J. G. W., \& Jonker, C. (2003). Similar network activated by young and old adults during the acquisition of a motor sequence. Neurobiology of Aging. https://doi.org/10.1016/S0197-4580(03)00030-7

Daw, N. D., Niv, Y., \& Dayan, P. (2005). Uncertainty-based competition between prefrontal and dorsolateral striatal systems for behavioral control. Nature Neuroscience. https://doi.org/10.1038/nn1560

Dayan, E., Censor, N., Buch, E. R., Sandrini, M., \& Cohen, L. G. (2013). Noninvasive brain stimulation: From physiology to network dynamics and back. In Nature Neuroscience. https://doi.org/10.1038/nn.3422

de Jesus, D. R., Favalli, G. P. de S., Hoppenbrouwers, S. S., Barr, M. S., Chen, R., Fitzgerald, P. B., \& Daskalakis, Z. J. (2014). Determining optimal rTMS parameters through changes in cortical inhibition. Clinical Neurophysiology. 
https://doi.org/10.1016/j.clinph.2013.09.011

De Vries, M. H., Barth, A. C. R., Maiworm, S., Knecht, S., Zwitserlood, P., \& Flöel, A. (2010). Electrical stimulation of Broca's area enhances implicit learning of an artificial grammar. Journal of Cognitive Neuroscience. https://doi.org/10.1162/jocn.2009.21385

De Witte, S., Klooster, D., Dedoncker, J., Duprat, R., Remue, J., \& Baeken, C. (2018). Left prefrontal neuronavigated electrode localization in tDCS: 10-20 EEG system versus MRIguided neuronavigation. Psychiatry Research - Neuroimaging. https://doi.org/10.1016/j.pscychresns.2018.02.001

Dirnberger, G., Novak, J., \& Nasel, C. (2013). Perceptual sequence learning is more severely impaired than motor sequence learning in patients with chronic cerebellar stroke. Journal of Cognitive Neuroscience. https://doi.org/10.1162/jocn_a_00444

Doyon, J., Gaudreau, D., Laforce, R. L., Castonguay, M., Bédard, P. J., Bédard, F., \& Bouchard, J. P. (1997). Role of the striatum, cerebellum, and frontal lobes in the learning of a visuomotor sequence. Brain and Cognition. https://doi.org/10.1006/brcg.1997.0899

Duecker, F., \& Sack, A. T. (2015). Rethinking the role of sham TMS. Frontiers in Psychology. https://doi.org/10.3389/fpsyg.2015.00210

Ferrari, C., Cattaneo, Z., Oldrati, V., Casiraghi, L., Castelli, F., D’Angelo, E., \& Vecchi, T. (2018). TMS over the Cerebellum Interferes with Short-term Memory of Visual Sequences. Scientific Reports. https://doi.org/10.1038/s41598-018-25151-y

Fitzgerald, P. B., Fountain, S., \& Daskalakis, Z. J. (2006). A comprehensive review of the effects of rTMS on motor cortical excitability and inhibition. In Clinical Neurophysiology. https://doi.org/10.1016/j.clinph.2006.06.712

Galea, J. M., Albert, N. B., Ditye, T., \& Miall, R. C. (2010). Disruption of the dorsolateral prefrontal cortex facilitates the consolidation of procedural skills. Journal of Cognitive Neuroscience. https://doi.org/10.1162/jocn.2009.21259

Gann, M. A., King, B. R., Dolfen, N., Veldman, M. P., Chan, K. L., Puts, N. A. J., Edden, R. A. E., Davare, M., Swinnen, S. P., Mantini, D., Robertson, E. M., \& Albouy, G. (2020). Hippocampal and striatal responses during motor learning are modulated by prefrontal cortex stimulation. In bioRxiv. https://doi.org/10.1101/2020.06.05.136531

Gann, M. A., King, B. R., Dolfen, N., Veldman, M. P., Chan, K. L., Puts, N. A. J., Edden, R. A. E., Davare, M., Swinnen, S. P., Mantini, D., Robertson, E. M., \& Albouy, G. (2021). Hippocampal and striatal responses during motor learning are modulated by prefrontal cortex stimulation. NeuroImage. https://doi.org/10.1016/j.neuroimage.2021.118158

Gentner, R., Wankerl, K., Reinsberger, C., Zeller, D., \& Classen, J. (2008). Depression of 
human corticospinal excitability induced by magnetic theta-burst stimulation: Evidence of rapid polarity-reversing metaplasticity. Cerebral Cortex. https://doi.org/10.1093/cercor/bhm239

Goldsworthy, M. R., Hordacre, B., Rothwell, J. C., \& Ridding, M. C. (2021). Effects of rTMS on the brain: is there value in variability? Cortex. https://doi.org/10.1016/j.cortex.2021.02.024

Gómez-Beldarrain, M., García-Moncó, J. C., Rubio, B., \& Pascual-Leone, A. (1998). Effect of focal cerebellar lesions on procedural learning in the serial reaction time task. Experimental Brain Research. https://doi.org/10.1007/s002210050374

Grafton, S. T., Hazeltine, E., \& Ivry, R. B. (1998). Abstract and effector-specific representations of motor sequences identified with pet. Journal of Neuroscience. https://doi.org/10.1523/jneurosci.18-22-09420.1998

Grafton, S. T., Hazeltine, E., \& Ivry, R. B. (2002). Motor sequence learning with the nondominant left hand: A PET functional imaging study. Experimental Brain Research. https://doi.org/10.1007/s00221-002-1181-y

Hamada, M., Murase, N., Hasan, A., Balaratnam, M., \& Rothwell, J. C. (2013). The role of interneuron networks in driving human motor cortical plasticity. Cerebral Cortex. https://doi.org/10.1093/cercor/bhs 147

Hazeltine, E., Grafton, S. T., \& Ivry, R. (1997). Attention and stimulus characteristics determine the locus of motor-sequence encoding. A PET study. Brain. https://doi.org/10.1093/brain/120.1.123

Hikosaka, O., Sakai, K., Lu, X., Nakahara, H., Rand, M. K., Nakamura, K., Miyachi, S., \& Doya, K. (1999). Parallel neural networks for learning sequential procedures. In Trends in Neurosciences. https://doi.org/10.1016/S0166-2236(99)01439-3

Howard, D. V., Howard, J. H., Japikse, K., DiYanni, C., Thompson, A., \& Somberg, R. (2004). Implicit Sequence Learning: Effects of Level of Structure, Adult Age, and Extended Practice. Psychology and Aging. https://doi.org/10.1037/0882-7974.19.1.79

Huang, Y. Z., Edwards, M. J., Rounis, E., Bhatia, K. P., \& Rothwell, J. C. (2005). Theta burst stimulation of the human motor cortex. Neuron. https://doi.org/10.1016/j.neuron.2004.12.033

Huang, Y. Z., Lu, M. K., Antal, A., Classen, J., Nitsche, M., Ziemann, U., Ridding, M., Hamada, M., Ugawa, Y., Jaberzadeh, S., Suppa, A., Paulus, W., \& Rothwell, J. (2017). Plasticity induced by non-invasive transcranial brain stimulation: A position paper. In Clinical Neurophysiology. https://doi.org/10.1016/j.clinph.2017.09.007 
Janacsek, K., \& Nemeth, D. (2013). Implicit sequence learning and working memory: Correlated or complicated? In Cortex. https://doi.org/10.1016/j.cortex.2013.02.012

Janacsek, K., \& Nemeth, D. (2015). The puzzle is complicated: When should working memory be related to implicit sequence learning, and when should it not? (Response to Martini etal.). In Cortex. https://doi.org/10.1016/j.cortex.2014.07.020

Janacsek, K., Shattuck, K. F., Tagarelli, K. M., Lum, J. A. G., Turkeltaub, P. E., \& Ullman, M. T. (2020). Sequence learning in the human brain: A functional neuroanatomical metaanalysis of serial reaction time studies. NeuroImage. https://doi.org/10.1016/j.neuroimage.2019.116387

Japikse, K. C., Negash, S., Howard, J. H., \& Howard, D. V. (2003). Intermanual transfer of procedural learning after extended practice of probabilistic sequences. Experimental Brain Research. https://doi.org/10.1007/s00221-002-1264-9

Keele, S. W., Mayr, U., Ivry, R., Hazeltine, E., \& Heuer, H. (2003). The Cognitive and Neural Architecture of Sequence Representation. In Psychological Review. https://doi.org/10.1037/0033-295X.110.2.316

Keramati, M., Dezfouli, A., \& Piray, P. (2011). Speed/accuracy trade-off between the habitual and the goal-directed processes. PLoS Computational Biology. https://doi.org/10.1371/journal.pcbi.1002055

Kim, Y. H., Park, J. W., Ko, M. H., Jang, S. H., \& Lee, P. K. W. (2004). Facilitative effect of high frequency subthreshold repetitive transcranial magnetic stimulation on complex sequential motor learning in humans. Neuroscience Letters. https://doi.org/10.1016/j.neulet.2004.05.113

Klomjai, W., Katz, R., \& Lackmy-Vallée, A. (2015). Basic principles of transcranial magnetic stimulation (TMS) and repetitive TMS (rTMS). In Annals of Physical and Rehabilitation Medicine. https://doi.org/10.1016/j.rehab.2015.05.005

Lang, N., Harms, J., Weyh, T., Lemon, R. N., Paulus, W., Rothwell, J. C., \& Siebner, H. R. (2006). Stimulus intensity and coil characteristics influence the efficacy of rTMS to suppress cortical excitability. Clinical Neurophysiology. https://doi.org/10.1016/j.clinph.2006.05.030

Lieberman, M. D. (2000). Intuition: A social cognitive neuroscience approach. Psychological Bulletin. https://doi.org/10.1037/0033-2909.126.1.109

Lum, J. A. G., Mills, A., Plumridge, J. M. A., Sloan, N. P., Clark, G. M., Hedenius, M., \& Enticott, P. G. (2018). Transcranial direct current stimulation enhances retention of a second (but not first) order conditional visuo-motor sequence. Brain and Cognition. 
https://doi.org/10.1016/j.bandc.2018.09.006

Maeda, F., Keenan, J. P., Tormos, J. M., Topka, H., \& Pascual-Leone, A. (2000). Interindividual variability of the modulatory effects of repetitive transcranial magnetic stimulation on cortical excitability. Experimental Brain Research. https://doi.org/10.1007/s002210000432

McCalley, D. M., Lench, D. H., Doolittle, J. D., Imperatore, J. P., Hoffman, M., \& Hanlon, C. A. (2021). Determining the optimal pulse number for theta burst induced change in cortical excitability. Scientific Reports. https://doi.org/10.1038/s41598-021-87916-2

Miller, E. K., \& Cohen, J. D. (2001). An integrative theory of prefrontal cortex function. In Annual Review of Neuroscience. https://doi.org/10.1146/annurev.neuro.24.1.167

Muellbacher, W., Zlemann, U., Wissel, J., Dang, N., Kofler, M., Facchini, S., Boroojerdi, B., Poewe, W., \& Hallett, M. (2002). Early consolidation in human primary motor cortex. Nature. https://doi.org/10.1038/nature712

Nemeth, D., Hallgató, E., Janacsek, K., Sándor, T., \& Londe, Z. (2009). Perceptual and motor factors of implicit skill learning. NeuroReport. https://doi.org/10.1097/WNR.0b013e328333ba08

Nemeth, D., Janacsek, K., Csifcsak, G., Szvoboda, G., Howard, J. H., \& Howard, D. V. (2011). Interference between sentence processing and probabilistic implicit sequence learning. PLoS ONE. https://doi.org/10.1371/journal.pone.0017577

Nissen, M. J., \& Bullemer, P. (1987). Attentional requirements of learning: Evidence from performance measures. Cognitive Psychology. https://doi.org/10.1016/00100285(87)90002-8

Pascual-Leone, A., Gates, J. R., \& Dhuna, A. (1991). Induction of speech arrest and counting errors with rapid-rate transcranial magnetic stimulation. Neurology. https://doi.org/10.1212/WNL.41.5.697

Pascual-Leone, A., Wassermann, E. M., Grafman, J., \& Hallett, M. (1996). The role of the dorsolateral prefrontal cortex in implicit procedural learning. Experimental Brain Research. https://doi.org/10.1007/BF00230427

Peigneux, P., Laureys, S., Delbeuck, X., \& Maquet, P. (2001). Sleeping brain, learning brain. the role of sleep for memory systems. In NeuroReport. https://doi.org/10.1097/00001756200112210-00001

Pell, G. S., Roth, Y., \& Zangen, A. (2011). Modulation of cortical excitability induced by repetitive transcranial magnetic stimulation: Influence of timing and geometrical parameters and underlying mechanisms. In Progress in Neurobiology. 
https://doi.org/10.1016/j.pneurobio.2010.10.003

Perez, M. A., Tanaka, S., Wise, S. P., Willingham, D. T., \& Cohen, L. G. (2008). Time-specific contribution of the supplementary motor area to intermanual transfer of procedural knowledge. Journal of Neuroscience. https://doi.org/10.1523/JNEUROSCI.3416-08.2008

Polanía, R., Nitsche, M. A., \& Ruff, C. C. (2018). Studying and modifying brain function with non-invasive brain stimulation. In Nature Neuroscience. https://doi.org/10.1038/s41593017-0054-4

Poldrack, R. A., Sabb, F. W., Foerde, K., Tom, S. M., Asarnow, R. F., Bookheimer, S. Y., \& Knowlton, B. J. (2005). The neural correlates of motor skill automaticity. Journal of Neuroscience. https://doi.org/10.1523/JNEUROSCI.3880-04.2005

Prashad, S., Du, Y., \& Clark, J. E. (2021). Sequence Structure Has a Differential Effect on Underlying Motor Learning Processes. Journal of Motor Learning and Development. https://doi.org/10.1123/jmld.2020-0031

Reber, A. S. (1967). Implicit learning of artificial grammars. Journal of Verbal Learning and Verbal Behavior. https://doi.org/10.1016/S0022-5371(67)80149-X

Reber, A. S. (1989). Implicit Learning and Tacit Knowledge. Journal of Experimental Psychology: General. https://doi.org/10.1037/0096-3445.118.3.219

Robertson. (2007). The serial reaction time task: Implicit motor skill learning? In Journal of Neuroscience. https://doi.org/10.1523/JNEUROSCI.2747-07.2007

Robertson, E. M., Tormos, J. M., Maeda, F., \& Pascual-Leone, A. (2001). The role of the dorsolateral prefrontal cortex during sequence learning is specific for spatial information. Cerebral Cortex. https://doi.org/10.1093/cercor/11.7.628

Robertson, Press, \& Pascual-Leone. (2005). Off-line learning and the primary motor cortex. Journal of Neuroscience. https://doi.org/10.1523/JNEUROSCI.1851-05.2005

Rosenthal, C. R., Roche-Kelly, E. E., Husain, M., \& Kennard, C. (2009). Response-dependent contributions of human primary motor cortex and angular gyrus to manual and perceptual sequence learning. Journal of Neuroscience. https://doi.org/10.1523/JNEUROSCI.260309.2009

Rothkegel, H., Sommer, M., \& Paulus, W. (2010). Breaks during 5 Hz rTMS are essential for facilitatory after effects. Clinical Neurophysiology. https://doi.org/10.1016/j.clinph.2009.11.016

Ruitenberg, M. F. L., Verwey, W. B., Schutter, D. J. L. G., \& Abrahamse, E. L. (2014). Cognitive and neural foundations of discrete sequence skill: A TMS study. Neuropsychologia. https://doi.org/10.1016/j.neuropsychologia.2014.01.014 
Sack, A. T., Camprodon, J. A., Pascual-Leone, A., \& Goebel, R. (2005). Neuroscience: The dynamics of interhemispheric compensatory processes in mental imagery. Science. https://doi.org/10.1126/science.1107784

Sack, Alexander T., Kadosh, R. C., Schuhmann, T., Moerel, M., Walsh, V., \& Goebel, R. (2009). Optimizing functional accuracy of TMS in cognitive studies: A comparison of methods. Journal of Cognitive Neuroscience. https://doi.org/10.1162/jocn.2009.21126

Seidler, R. D., Purushotham, A., Kim, S. G., Ugurbil, K., Willingham, D., \& Ashe, J. (2005). Neural correlates of encoding and expression in implicit sequence learning. Experimental Brain Research. https://doi.org/10.1007/s00221-005-2284-z

Seidter, R. D., Purushotham, A., Kim, S. G., Uğurbil, K., Willingham, D., \& Ashe, J. (2002). Cerebellum activation associated with performance change but not motor learning. Science. https://doi.org/10.1126/science.1068524

Shanks, D. R. (2005). Implicit learning. In Handbook of Cognition. https://doi.org/10.4135/9781848608177.n8

Shin, J. C., \& Ivry, R. B. (2003). Spatial and Temporal Sequence Learning in Patients with Parkinson's Disease or Cerebellar Lesions. Journal of Cognitive Neuroscience. https://doi.org/10.1162/089892903322598175

Smalle, E. H. M., Panouilleres, M., Szmalec, A., \& Möttönen, R. (2017). Language learning in the adult brain: Disrupting the dorsolateral prefrontal cortex facilitates word-form learning. Scientific Reports. https://doi.org/10.1038/s41598-017-14547-x

Song, S., Howard, J. H., \& Howard, D. V. (2007). Sleep does not benefit probabilistic motor sequence learning. Journal of Neuroscience. https://doi.org/10.1523/JNEUROSCI.206207.2007

Song, S., Howard, J. H., \& Howard, D. V. (2008). Perceptual sequence learning in a serial reaction time task. Experimental Brain Research. https://doi.org/10.1007/s00221-0081411-z

Steel, A., Song, S., Bageac, D., Knutson, K. M., Keisler, A., Saad, Z. S., Gotts, S. J., Wassermann, E. M., \& Wilkinson, L. (2016). Shifts in connectivity during procedural learning after motor cortex stimulation: A combined transcranial magnetic stimulation/functional magnetic resonance imaging study. Cortex. https://doi.org/10.1016/j.cortex.2015.10.004

Thut, G., \& Pascual-Leone, A. (2010). A review of combined TMS-EEG studies to characterize lasting effects of repetitive TMS and assess their usefulness in cognitive and clinical neuroscience. In Brain Topography. https://doi.org/10.1007/s10548-009-0115-4 
Torriero, S., Oliveri, M., Koch, G., Caltagirone, C., \& Petrosini, L. (2004). Interference of left and right cerebellar rTMS with procedural learning. Journal of Cognitive Neuroscience. https://doi.org/10.1162/0898929042568488

Tunovic, S., Press, D. Z., \& Robertson, E. M. (2014). A physiological signal that prevents motor skill improvements during consolidation. Journal of Neuroscience. https://doi.org/10.1523/JNEUROSCI.3497-13.2014

Turi, Z., Lenz, M., Paulus, W., Mittner, M., \& Vlachos, A. (2021). Selecting stimulation intensity in repetitive transcranial magnetic stimulation studies: A systematic review between 1991 and 2020. In European Journal of Neuroscience. https://doi.org/10.1111/ejn.15195

Uddén, J., Ingvar, M., Hagoort, P., \& Petersson, K. M. (2017). Broca’s region: A causal role in implicit processing of grammars with crossed non-adjacent dependencies. Cognition. https://doi.org/10.1016/j.cognition.2017.03.010

van der Graaf, F. H. C. E., Maguire, R. P., Leenders, K. L., \& de Jong, B. M. (2006). Cerebral activation related to implicit sequence learning in a Double Serial Reaction Time task. Brain Research. https://doi.org/10.1016/j.brainres.2006.01.103

Veldman, M. P., Maurits, N. M., Nijland, M. A. M., Wolters, N. E., Mizelle, J. C., \& Hortobágyi, T. (2018). Spectral and temporal electroencephalography measures reveal distinct neural networks for the acquisition, consolidation, and interlimb transfer of motor skills in healthy young adults. Clinical Neurophysiology. https://doi.org/10.1016/j.clinph.2017.12.003

Verwey, W. B., Lammens, R., \& Van Honk, J. (2002). On the role of the SMA in the discrete sequence production task: A TMS study. Neuropsychologia. https://doi.org/10.1016/S0028-3932(01)00221-4

Walker, M. P., \& Stickgold, R. (2005). It's practice, with sleep, that makes perfect: Implications of sleep-dependent learning and plasticity for skill performance. In Clinics in Sports Medicine. https://doi.org/10.1016/j.csm.2004.11.002

Wan Lee, S., Shimojo, S., \& O’Doherty, J. P. (2014). Neural Computations Underlying Arbitration between Model-Based and Model-free Learning. Neuron. https://doi.org/10.1016/j.neuron.2013.11.028

Wilkinson, L., Steel, A., Mooshagian, E., Zimmermann, T., Keisler, A., Lewis, J. D., \& Wassermann, E. M. (2015). Online feedback enhances early consolidation of motor sequence learning and reverses recall deficit from transcranial stimulation of motor cortex. Cortex. https://doi.org/10.1016/j.cortex.2015.06.012 
Wilkinson, L., Teo, J. T., Obeso, I., Rothwell, J. C., \& Jahanshahi, M. (2010). The contribution of primary motor cortex is essential for probabilistic implicit sequence learning: Evidence from theta burst magnetic stimulation. Journal of Cognitive Neuroscience. https://doi.org/10.1162/jocn.2009.21208

Willingham, D. B., Salidis, J., \& Gabrieli, J. D. E. (2002). Direct comparison of neural systems mediating conscious and unconscious skill learning. Journal of Neurophysiology. https://doi.org/10.1152/jn.2002.88.3.1451

Yuan, P., \& Raz, N. (2014). Prefrontal cortex and executive functions in healthy adults: A metaanalysis of structural neuroimaging studies. In Neuroscience and Biobehavioral Reviews. https://doi.org/10.1016/j.neubiorev.2014.02.005 